Noname manuscript No.

(will be inserted by the editor)

\title{
The Gradient Superconvergence of Bilinear Finite Volume Element for Elliptic Problems
}

\author{
Tie Zhang · Lixin Tang
}

Received: date / Accepted: date

\begin{abstract}
We study the gradient superconvergence of bilinear finite volume element (FVE) solving the elliptic problems. First, a superclose weak estimate is established for the bilinear form of the FVE method. Then, we prove that the gradient approximation of the FVE solution has the superconvergence property: $\max _{P \in S}\left|\left(\nabla u-\bar{\nabla} u_{h}\right)(P)\right|=$ $O\left(h^{2}\right)|\ln h|$, where $\bar{\nabla} u_{h}(P)$ denotes the average gradient on elements containing point $P$ and $S$ is the set of optimal stress points composed of the mesh points, the midpoints of edges and elements.
\end{abstract}

Keywords Bilinear finite volume element · elliptic problem · gradient approximation $\cdot$ superconvergence

This work was supported by the National Natural Science Funds of China, No. 11371081; and the State Key Laboratory of Synthetical Automation for Process Industries Fundamental Research Funds, No. $2013 \mathrm{ZCX} 02$.

T. Zhang (Corresponding author)

Department of Mathematics and the State Key Laboratory of Synthetical Automation for Process Industries, Northeastern University, Shenyang 110004, China

E-mail: ztmath@163.com Tel.: +086-24-83680949 Fax: +086-24-83680949

L.X. Tang

The State Key Laboratory of Synthetical Automation for Process Industries, Northeastern University, Shenyang 110004, China

E-mail: lixintang@mail.neu.edu.cn 
Mathematics Subject Classification (2010) $65 \mathrm{~N} 15 \cdot 65 \mathrm{~N} 30 \cdot 65 \mathrm{M} 60$

\section{Introduction}

The finite volume element (FVE) method has been widely used in numerically solving partial deferential equations. The main feature of FVE method is that it inherits some physical conservation laws of original problems locally, which are very desirable in practical applications. During the last decades, many research works have been presented for FVE methods solving various partial differential equations, see $[1,2,3,4,5,6,7,8,9,10,12,13,14,15,17,18,20]$ and the references cited therein.

Superconvergence of numerical solutions has been an active research area for finite element method (FEM) since its practical importance in enhancing the accuracy of finite element approximation. But the study of superconvergence properties of FVE methods is far behind that of FEMs. For elliptic problems in two-dimensional domain, the early superconvergence results of linear and bilinear FVE solutions are of this form [3, 10]

$$
\left(\frac{1}{N} \sum_{z \in S}\left|\left(\nabla u-\bar{\nabla} u_{h}\right)(z)\right|^{2}\right)^{\frac{1}{2}} \leq C h^{2}\|u\|_{3, \infty},
$$

where $S$ is the set of optimal stress points of interpolation function on partition $T_{h}$, $N=O\left(h^{-2}\right)$ is the total number of points in $S$, and $\bar{\nabla}$ denotes the average gradient on elements containing point $z$. Later, $\mathrm{Lv}$ and $\mathrm{Li}$ in [12] extended result (1.1) to the isoparametric bilinear FVE on quadrilateral meshes under the $h^{2}$-uniform mesh condition. Recently, Zhang and Zou in [20] also derived some superconvergence results for the bi-complete $k$-order FVE on rectangular meshes, and in the case of bilinear $\operatorname{FVE}(k=1)$, their result is

$$
\left|\nabla\left(u-u_{h}\right)(G)\right| \leq C h^{2}|\ln h|^{\frac{1}{2}}\|u\|_{4, \infty}
$$

where $G$ is the Gauss point of element (the midpoint of element). Moreover, by using the postprocessing technique, Chou and Ye [7] obtain the superconvergence estimate:

$$
\left.\| \nabla u-\nabla Q u_{h}\right)\left\|\leq C h^{\frac{3}{2}}\right\| u \|_{3}
$$


where $Q u_{h}$ is the postprocessed linear FVE solution obtained by the $L_{2}$-projection method; Zhang and Sheng [18] further derive the superconvergence estimate:

$$
\left.\| \nabla u-R \nabla u_{h}\right)\left\|\leq C h^{2}\right\| u \|_{3}
$$

where $R \nabla u_{h}$ is the reconstructed gradient of bilinear FVE solution obtained by using the patch interpolation recovery method.

In this paper, we consider the bilinear FVE method to solve the following problem

$$
\left\{\begin{array}{l}
-\operatorname{div}(A \nabla u)+c u=f, \text { in } \Omega, \\
u=0, \text { on } \partial \Omega
\end{array}\right.
$$

where $\Omega \subset R^{2}$ is a rectangular domain with boundary $\partial \Omega$, coefficient matrix $A=$ $\left(a_{i j}\right)_{2 \times 2}$. Our main goal is to give some piecewise-point gradient superconvergence for the bilinear FVE approximation to problem (1.5). To the authors' best knowledge, in existing literatures, only the midpoints of elements are proved to be the superconvergence point of gradient approximation [20] (also see (1.2)), we here will prove that except the midpoints of elements, all interior mesh points and midpoints of interior edges are also the superconvergence points. Generally speaking, the analysis of bilinear FVE on rectangular meshes is more difficult then that of linear FVE on triangle meshes, the reason is that $\nabla u_{h}$ is not constant in the former case. By calculating exactly some integrals on element and its boundary, we first establish the superclose weak estimate for the bilinear form of the FVE method,

$$
\left|a_{h}\left(u-\Pi_{h} u, \Pi_{h}^{*} v\right)\right| \leq C h^{2}\|u\|_{3, p}\|v\|_{1, q}, \forall v \in U_{h}, 2 \leq p \leq \infty, 1 / p+1 / q=1
$$

where $\Pi_{h}$ is the bilinear interpolation operator, $\Pi_{h}^{*}$ is the projection operator from the trial function space $U_{h}$ to the test function space. It is well known that such weak estimate plays an important role in the superconvergence analysis of FEMs [11, 19, 21]. By means of this weak estimate and some integral estimates on element, we further derive the following superconvergence result for the gradient approximation

$$
\max _{P \in S}\left|\left(\nabla u-\bar{\nabla} u_{h}\right)(P)\right| \leq C h^{2}|\ln h|\|u\|_{3, \infty},
$$


where $S$ is the optimal stress point set of bilinear interpolation function which is composed of the interior mesh points, the midpoints of interior edges and the midpoints of elements.

This paper is organized as follows. In Section 2, we introduce the FVE method and give some lemmas. In Section 3, we establish the superconvergence weak estimate. Section 4 is devoted to the piecewise-point gradient supconvergence estimate on the set $S$. Finally, in Section 5, numerical experiments are provided to illustrate our theoretical analysis.

Throughout this paper, we adopt the notations $W^{m, p}(D)$ to indicate the usual Sobolev spaces on domain $D \subset \Omega$ equipped with the norm $\|\cdot\|_{m, p, D}$ and semi-norm $|\cdot|_{m, p, D}$, and if $p=2$, we set $W^{m, p}(D)=H^{m}(D),\|\cdot\|_{m, p, D}=\|\cdot\|_{m, D}$. When $D=\Omega$, we omit the index $D$. We will use letter $C$ to represent a generic positive constant, independent of the mesh size $h$.

\section{Finite volume element method}

Consider problem (1.5). As usual, we assume that there exist positive constants $C_{1}$ and $C_{2}$ such that

$$
C_{1} \xi^{T} \xi \leq \xi^{T} A(x, y) \xi \leq C_{2} \xi^{T} \xi, \forall \xi \in R^{2},(x, y) \in \Omega
$$

We further assume that $A \in\left[W^{1, \infty}(\Omega)\right]^{2 \times 2}, c \in L_{\infty}(\Omega)$ and $c \geq 0$.

Let $T_{h}=\bigcup\{K\}$ be a rectangular partition of domain $\Omega$ so that $\bar{\Omega}=\bigcup_{K \in T_{h}}\{\bar{K}\}$, where $h=\operatorname{maxh}_{K}, h_{K}$ is the diameter of element $K$. We assume that partition $T_{h}$ is regular, that is, there exists a positive constant $\gamma>0$ such that

$$
h_{K} / \rho_{K} \leq \gamma, \forall K \in T_{h},
$$

where $\rho_{K}$ denotes the diameter of the biggest ball included in $K$.

Associated with partition $T_{h}$, we construct the central dual partition $T_{h}^{*}$ by connecting the center of each element to the midpoints of edges by straight lines. Thus, for each nodal point $P$ in $T_{h}$, there exists a rectangle $K_{P}^{*}$ surrounding $P, K_{P}^{*} \in T_{h}^{*}$ is 
called the dual element or the control volume at point $P$, see Fig.1.

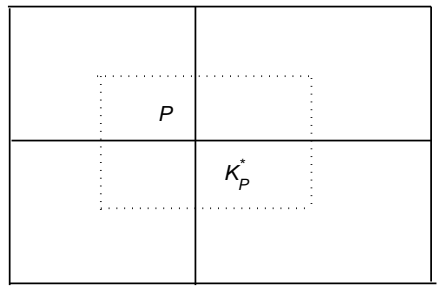

FIG.1. The dual element $K_{P}^{*}$ surrounding point $P$

To partition $T_{h}$ and $T_{h}^{*}$, we associate the following trial function space $U_{h}$ and test function space $V_{h}$, respectively,

$$
\begin{aligned}
& U_{h}=\left\{u_{h} \in C^{0}(\bar{\Omega}):\left.u_{h}\right|_{K} \in Q_{1}(K), \forall K \in T_{h},\left.u_{h}\right|_{\partial \Omega}=0\right\}, \\
& V_{h}=\left\{v_{h} \in L_{2}(\Omega):\left.v_{h}\right|_{K_{P}^{*}}=\text { constant }, \forall P \in N_{h},\left.v_{h}\right|_{K_{P}^{*}}=0, \forall P \in \partial \Omega\right\},
\end{aligned}
$$

where $Q_{1}(K)$ is the set of all bilinear polynomials on $K$ and $N_{h}$ is the set of all nodal points of $T_{h}$

The conventional weak form for problem (1.5) is that find $u \in H_{0}^{1}(\Omega)$ such that

$$
a(u, v)=(f, v), \forall v \in H_{0}^{1}(\Omega),
$$

where

$$
a(u, v)=\int_{\Omega} A \nabla u \cdot \nabla v+c u v,(f, v)=\int_{\Omega} f v .
$$

Weak form (2.3) usually is adopted for the finite element method. But for the FVE method, we need a new weak form. Let $u$ be the solution of problem (1.5), then by using Green's formula, we have

$$
-\int_{\partial K_{P}^{*}} n \cdot(A \nabla u) v d s+\int_{K_{P}^{*}} c u v=\int_{K_{P}^{*}} f v, K_{P}^{*} \in T_{h}^{*}, v \in V_{h},
$$

where $n$ is the outward unit normal vector on the boundary concerned. Motivated by this weak formula, we introduce the following bilinear form

$$
a_{h}(u, v)=\sum_{K_{P}^{*} \in T_{h}^{*}}\left(-\int_{\partial K_{P}^{*}} n \cdot(A \nabla u) v d s+\int_{K_{P}^{*}} c u v\right), u \in H^{1}(\Omega), v \in V_{h},
$$

and define the FVE approximation of problem (1.5) by finding $u_{h} \in U_{h}$ such that

$$
a_{h}\left(u_{h}, v_{h}\right)=\left(f, v_{h}\right), \forall v_{h} \in V_{h} .
$$


Let $\Pi_{h}^{*}: U_{h} \rightarrow V_{h}$ be the interpolation operator defined by

$$
\Pi_{h}^{*} v_{h}=\sum_{P \in N_{h}} v_{h}(P) \chi_{P}, \forall v_{h} \in U_{h}
$$

where $\chi_{P}$ is the characteristic function of the dual element $K_{P}^{*}$. Since $\Pi_{h}^{*}$ is a one to one mapping from $U_{h}$ onto $V_{h}$, then the equivalent form of problem (2.7) is that find $u_{h} \in U_{h}$ such that

$$
a_{h}\left(u_{h}, \Pi_{h}^{*} v_{h}\right)=\left(f, \Pi_{h}^{*} v_{h}\right), \forall v_{h} \in U_{h}
$$

This is the FVE scheme to be used in our analysis. From 2.5) we know that scheme (2.8) is consistent and the following error equation holds.

$$
a_{h}\left(u-u_{h}, \Pi_{h}^{*} v_{h}\right)=0, \forall v_{h} \in U_{h}
$$

Let $\Pi_{h} u$ be the usual bilinear interpolation function of continuous function $u$. In our analysis, the following approximation property, trace inequality and inverse inequality will be used frequently. For $1 \leq p, q \leq \infty$, we have

$$
\begin{aligned}
& \left\|u-\Pi_{h} u\right\|_{m, p, K} \leq C h_{K}^{2-m}\|u\|_{2, p, K}, 0 \leq m \leq 2,1<p \leq \infty, \\
& \|u\|_{0, p, \partial K} \leq C h_{K}^{-\frac{1}{p}}\left(\|u\|_{0, p, K}+h_{K}\|\nabla u\|_{0, p, K}\right), u \in W^{1, p}(K), \\
& \left\|u_{h}\right\|_{m, p, K} \leq C h_{K}^{\frac{2}{p}-\frac{2}{q}} h_{K}^{l-m}\left\|u_{h}\right\|_{l, q, K}, u_{h} \in Q_{1}(K), 0 \leq l \leq m \leq 1 .
\end{aligned}
$$

Furthermore, the following two lemmas hold [10, 18].

Lemma 2.1 Let $K \in T_{h}, \tau \subset \partial K$ be an edge of $K$. Then, for $v_{h} \in U_{h}, 1 \leq q \leq \infty$, we have

$$
\begin{aligned}
& \int_{K}\left(v_{h}-\Pi_{h}^{*} v_{h}\right)=0, \quad \int_{\tau}\left(v_{h}-\Pi_{h}^{*} v_{h}\right) d s=0, \\
& \left\|v_{h}-\Pi_{h}^{*} v_{h}\right\|_{0, q, K} \leq C h_{K}\left\|v_{h}\right\|_{1, q, K}, \\
& \left\|v_{h}-\Pi_{h}^{*} v_{h}\right\|_{0, q, \partial K} \leq C h_{K}^{1-\frac{1}{q}}\left\|v_{h}\right\|_{1, q, K} .
\end{aligned}
$$

Lemma 2.2 For h small, we have

$$
a_{h}\left(v_{h}, \Pi_{h}^{*} v_{h}\right) \geq C\left\|v_{h}\right\|_{1}^{2}, \forall v_{h} \in U_{h} .
$$

Lemma 2.2 implies that the solution $u_{h}$ of problem 2.8 uniquely exists. 


\section{Interpolation weak estimate}

It is well known that the interpolation weak estimate plays an important role in the superconvergence analysis [11,19,21]. To FEM defined on regular rectangular meshes, the following interpolation weak estimate has been established [19,21]

$$
\left|a\left(u-\Pi_{h} u, v\right)\right| \leq C h^{2}\|u\|_{3, p}\|v\|_{1, q}, \forall v \in U_{h}, 2 \leq p \leq \infty, 1 / p+1 / q=1 .
$$

In this section, we will give a similar weak estimate for the FVE method, which is very useful in our superconvergence analysis.

In order to utilize the known result (3.1), we need to give the difference between the bilinear form of FVE and that of FEM.

Lemma 3.1 For any $w \in U_{h}+H^{2}(\Omega), v_{h} \in U_{h}$, we have

$$
\begin{aligned}
a_{h}\left(w, \Pi_{h}^{*} v_{h}\right)-a\left(w, v_{h}\right) & =\sum_{K \in T_{h}} \int_{\partial K} n \cdot(A \nabla w)\left(\Pi_{h}^{*} v_{h}-v_{h}\right) d s \\
& +\sum_{K \in T_{h}}\left(-\operatorname{div}(A \nabla w)+c w, \Pi_{h}^{*} v_{h}-v_{h}\right)_{K}
\end{aligned}
$$

where $U_{h}+H^{2}(\Omega)=\left\{w=u_{h}+v: u_{h} \in U_{h}, v \in H^{2}(\Omega)\right\}$ is the the algebraic sum space.

Proof By Green's formula, we have

$$
\int_{K} A \nabla w \cdot \nabla v_{h}=-\int_{K} \operatorname{div}(A \nabla w) v_{h}+\int_{\partial K} n \cdot(A \nabla w) v_{h} d s
$$

and (see Fig.1)

$$
\begin{aligned}
& \sum_{K \in T_{h}} \int_{K} \operatorname{div}(A \nabla w) \Pi_{h}^{*} v_{h}=\sum_{K \in T_{h}} \sum_{P} \in T_{h}^{*} \int_{K_{P}^{*} \cap K} \operatorname{div}(A \nabla w) \Pi_{h}^{*} v_{h} \\
= & \sum_{K \in T_{h}} \int_{\partial K} n \cdot(A \nabla w) \Pi_{h}^{*} v_{h} d s+\sum_{K_{P}^{*} \in T_{h}^{*}} \int_{\partial K_{P}^{*}} n \cdot(A \nabla w) \Pi_{h}^{*} v_{h} d s .
\end{aligned}
$$

Substituting this two identities into the definitions of $a\left(w, v_{h}\right)$ and $a_{h}\left(w, \Pi_{h}^{*} v_{h}\right)$ (see (2.4) and (2.6), the proof is completed.

Let $K=\square P_{1} P_{2} P_{3} P_{4}$ be a rectangular element, $h_{x}=x_{2}-x_{1}, h_{y}=y_{2}-y_{1}$, see Fig.2. The following lemma gives some exact calculations for integrals on $\partial K$. 


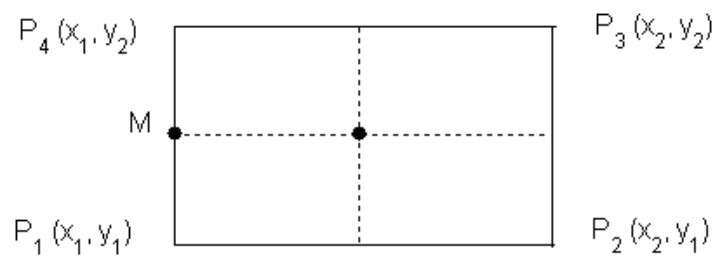

FIG.2. Rectangular element $K=\square P_{1} P_{2} P_{3} P_{4}$

Lemma 3.2 For $w, v \in U_{h}$, we have

$$
\begin{aligned}
& \int_{P_{1} P_{4}}\left(\Pi_{h}^{*} v-v\right) w_{x} d y=\frac{h_{y}^{3}}{24} v_{y}\left(x_{1}, y\right) w_{x y}, \quad \int_{P_{1} P_{4}}\left(\Pi_{h}^{*} v-v\right) w_{y} d y=0, \\
& \int_{P_{2} P_{3}}\left(\Pi_{h}^{*} v-v\right) w_{x} d y=\frac{h_{y}^{3}}{24} v_{y}\left(x_{2}, y\right) w_{x y}, \quad \int_{P_{2} P_{3}}\left(\Pi_{h}^{*} v-v\right) w_{y} d y=0, \\
& \int_{P_{1} P_{2}}\left(\Pi_{h}^{*} v-v\right) w_{y} d x=\frac{h_{x}^{3}}{24} v_{x}\left(x, y_{1}\right) w_{x y}, \quad \int_{P_{1} P_{2}}\left(\Pi_{h}^{*} v-v\right) w_{x} d x=0, \\
& \int_{P_{4} P_{3}}\left(\Pi_{h}^{*} v-v\right) w_{y} d x=\frac{h_{x}^{3}}{24} v_{x}\left(x, y_{2}\right) w_{x y}, \int_{P_{4} P_{3}}\left(\Pi_{h}^{*} v-v\right) w_{x} d x=0 .
\end{aligned}
$$

Proof We only prove (3.3), the other are similar. Noting that $w_{x}$ and $v$ are linear on line segment $P_{1} P_{4}$ ( $M$ is its middle point), we have from the definition of $\Pi_{h}^{*} v$ that

$$
\begin{aligned}
\int_{P_{1} P_{4}} \Pi_{h}^{*} v w_{x} d y & =\int_{P_{1} M} v\left(P_{1}\right) w_{x} d y+\int_{M P_{4}} v\left(P_{4}\right) w_{x} d y \\
& =\frac{h_{y}}{4} v\left(P_{1}\right)\left(w_{x}\left(P_{1}\right)+w_{x}(M)\right)+\frac{h_{y}}{4} v\left(P_{4}\right)\left(w_{x}(M)+w_{x}\left(P_{4}\right)\right) \\
& =\frac{h_{y}}{4}\left(v\left(P_{1}\right) w_{x}\left(P_{1}\right)+2 v(M) w_{x}(M)+v\left(P_{4}\right) w_{x}\left(P_{4}\right)\right) .
\end{aligned}
$$

On the other hand, using the composite trapezoidal rule:

$$
\int_{a}^{b} f(y) d y=\frac{b-a}{4}\left(f(a)+2 f\left(\frac{a+b}{2}\right)+f(b)\right)-\frac{(b-a)^{3}}{48} f_{y}^{\prime \prime}
$$

we obtain by taking $f=v w_{x}$ that

$$
\int_{P_{1} P_{4}} v w_{x} d y=\frac{h_{y}}{4}\left(v\left(P_{1}\right) w_{x}\left(P_{1}\right)+2 v(M) w_{x}(M)+v\left(P_{4}\right) w_{x}\left(P_{4}\right)\right)-\frac{h_{y}^{3}}{24} v_{y}\left(x_{1}, y\right) w_{x y} .
$$

The difference of above two equalities implies the first equality in (3.3). The second equality comes from (2.13), noting that $w_{y}$ is constant on $P_{1} P_{4}$. 
Let $w^{c}$ denote the piecewise constant approximation of function $w$ on $T_{h}$,

$$
\left.w^{c}\right|_{K}=\frac{1}{K} \int_{K} w, K \in T_{h} ;\left\|w-w^{c}\right\|_{0, p, K} \leq C h_{K}\|w\|_{1, p, K}, 1 \leq p \leq \infty
$$

Theorem 3.1 Let $T_{h}$ be a regular rectangular mesh and $u \in W^{3, p}(\Omega)$. Then we have

$$
\left|a_{h}\left(u-\Pi_{h} u, \Pi_{h}^{*} v\right)\right| \leq C h^{2}\|u\|_{3, p}\|v\|_{1, q}, \forall v \in U_{h}, 2 \leq p \leq \infty, 1 / p+1 / q=1 .
$$

Proof Let $A_{M}=\left(\bar{a}_{i j}\right)_{2 \times 2}$ be the value of $A$ at the midpoint $M$ of edge $\tau \subset \partial K$, see Fig.2. From Lemma 3.1, we have

$$
\begin{aligned}
& a_{h}\left(u-\Pi_{h} u, \Pi_{h}^{*} v\right)-a\left(u-\Pi_{h} u, v\right) \\
= & \sum_{K \in T_{h}} \int_{\partial K} n \cdot\left(A-A_{M}\right) \nabla\left(u-\Pi_{h} u\right)\left(\Pi_{h}^{*} v-v\right) d s \\
& +\sum_{K \in T_{h}} \int_{\partial K} n \cdot\left(A_{M} \nabla\left(u-\Pi_{h} u\right)\right)\left(\Pi_{h}^{*} v-v\right) d s \\
& +\sum_{K \in T_{h}}\left(-\operatorname{div}\left(A \nabla\left(u-\Pi_{h} u\right)\right), \Pi_{h}^{*} v-v\right)_{K} \\
& +\sum_{K \in T_{h}}\left(c\left(u-\Pi_{h} u\right), \Pi_{h}^{*} v-v\right)_{K} \\
= & E_{1}+E_{2}+E_{3}+E_{4} .
\end{aligned}
$$

Using (2.10)-2.11) and (2.14)-2.15), we obtain

$$
\begin{aligned}
& E_{1} \leq C \sum_{K \in T_{h}} h_{K}|A|_{1, \infty}\left\|\nabla\left(u-\Pi_{h} u\right)\right\|_{0, p, \partial K}\left\|v-\Pi_{h}^{*} v\right\|_{0, q, \partial K} \leq C h^{2}\|u\|_{2, p}\|v\|_{1, q} . \\
& E_{4} \leq C \sum_{K \in T_{h}}\left\|u-\Pi_{h} u\right\|_{1, p, K}\left\|v-\Pi_{h}^{*} v\right\|_{0, q, K} \leq C h^{2}\|u\|_{2, p}\|v\|_{1, q} .
\end{aligned}
$$

For $E_{3}$, set $A=\left(\mathbf{a}_{1}, \mathbf{a}_{2}\right)$. Since

$$
\operatorname{div}(A \nabla w)=\left(\operatorname{div} \mathbf{a}_{1}, \operatorname{div} \mathbf{a}_{2}\right) \cdot \nabla w+\mathbf{a}_{1} \cdot \nabla w_{x}+\mathbf{a}_{2} \cdot \nabla w_{y},
$$

then we have

$$
\begin{aligned}
E_{3}= & \sum_{K \in T_{h}}\left(-\left(\operatorname{div} \mathbf{a}_{1}, \operatorname{div} \mathbf{a}_{2}\right) \cdot \nabla\left(u-\Pi_{h} u\right), \Pi_{h}^{*} v-v\right)_{K} \\
& -\sum_{K \in T_{h}}\left(\mathbf{a}_{1} \cdot \nabla\left(u-\Pi_{h} u\right)_{x}+\mathbf{a}_{2} \cdot \nabla\left(u-\Pi_{h} u\right)_{y}, \Pi_{h}^{*} v-v\right)_{K}=E_{31}+E_{32} .
\end{aligned}
$$


Obviously, $E_{31} \leq C h^{2}\|u\|_{2, p}\|v\|_{1, q}$. Now, let $u_{i}=u\left(P_{i}\right), \xi=\left(x-x_{1}\right) / h_{x}, \eta=(y-$ $\left.y_{1}\right) / h_{y}$, see Fig.2. Since

$$
\Pi_{h} u=u_{1}+\left(u_{2}-u_{1}\right) \xi+\left(u_{4}-u_{1}\right) \eta+\left(u_{3}+u_{1}-u_{2}-u_{4}\right) \xi \eta,(x, y) \in K,
$$

hence

$$
\begin{aligned}
\left(\Pi_{h} u\right)_{x y} & =\left(u_{3}+u_{1}-u_{2}-u_{4}\right) h_{x}^{-1} h_{y}^{-1} \\
& =\frac{1}{K}\left(\int_{x_{1}}^{x_{2}} u_{x}\left(x, y_{2}\right) d x-\int_{x_{1}}^{x_{2}} u_{x}\left(x, y_{1}\right) d x\right) \\
& =\frac{1}{K} \int_{x_{1}}^{x_{2}} \int_{y_{1}}^{y_{2}} u_{x y} d x d y=\frac{1}{K} \int_{K} u_{x y} d x d y=\left(u_{x y}\right)^{c} .
\end{aligned}
$$

Therefore, we have from (2.13) that

$$
\begin{aligned}
E_{32}= & -\sum_{K \in T_{h}}\left(a_{11} u_{x x}+a_{21}\left(u_{x y}-\left(\Pi_{h} u\right)_{x y}\right)+a_{12}\left(u_{x y}-\left(\Pi_{h} u\right)_{x y}\right)+a_{22} u_{y y}, \Pi_{h}^{*} v-v\right)_{K} \\
= & -\sum_{K \in T_{h}}\left(a_{11} u_{x x}+a_{21}\left(u_{x y}-u_{x y}^{c}\right)+a_{12}\left(u_{x y}-u_{x y}^{c}\right)+a_{22} u_{y y}, \Pi_{h}^{*} v-v\right)_{K} \\
= & -\sum_{K \in T_{h}}\left(a_{11} u_{x x}+a_{22} u_{y y}-\left(a_{11} u_{x x}+a_{22} u_{y y}\right)^{c}, \Pi_{h}^{*} v-v\right)_{K} \\
& -\sum_{K \in T_{h}}\left(a_{21}\left(u_{x y}-u_{x y}^{c}\right)+a_{12}\left(u_{x y}-u_{x y}^{c}\right), \Pi_{h}^{*} v-v\right)_{K} .
\end{aligned}
$$

Using the approximation property, we obtain $E_{32} \leq C h^{2}\|u\|_{3, p}\|v\|_{1, q}$ so that

$$
E_{3}=E_{31}+E_{32} \leq C h^{2}\|u\|_{3, p}\|v\|_{1, q}
$$

Now we need to estimate

$$
E_{2}=\sum_{K \in T_{h}} \sum_{\tau \subset \partial K \backslash \partial \Omega} \int_{\tau} n \cdot\left(A_{M} \nabla\left(u-\Pi_{h} u\right)\right)\left(\Pi_{h}^{*} v-v\right) d s,
$$

noting that $\left.\left(\Pi_{h}^{*} v-v\right)\right|_{\partial \Omega}=0$. Let $\tau$ be an interior edge, that is, a common edge of two adjacent element $K$ and $K^{\prime}$. Since $\left.n\right|_{\tau \cap \partial K}=-\left.n\right|_{\tau \cap \partial K^{\prime}}$ and $A_{M} \nabla u\left(\Pi_{h}^{*} v-v\right)$ is continuous across edge $\tau$, then we have

$$
E_{2}=\sum_{K \in T_{h}} \sum_{\tau \subset \partial K \backslash \partial \Omega} \int_{\tau}-n \cdot\left(A_{M} \nabla \Pi_{h} u\right)\left(\Pi_{h}^{*} v-v\right) d s \equiv \sum_{K \in T_{h}} \sum_{\tau \subset \partial K \backslash \partial \Omega} F(\tau) .
$$


Without loss of generality, let $K=\square P_{1} P_{2} P_{3} P_{4}=\left(x_{1}, x_{2}\right) \times\left(y_{1}, y_{2}\right)$ be a rectangular element and the common edge $\tau=\partial K \bigcap \partial K^{\prime}=P_{1} P_{4}$, see Fig.2. From Lemma 3.2 we have

$$
F(\tau)=\int_{P_{1} P_{4}}\left(\bar{a}_{11}\left(\Pi_{h} u\right)_{x}+\bar{a}_{12}\left(\Pi_{h} u\right)_{y}\right)\left(\Pi_{h}^{*} v-v\right) d y=\bar{a}_{11} \frac{h_{y}^{3}}{24} v_{y}\left(x_{1}, y\right)\left(\Pi_{h} u\right)_{x y} .
$$

Then, from (3.14), (3.4) and (3.11), and noting that $\left.n\right|_{\tau \cap \partial K}=-\left.n\right|_{\tau \cap \partial K^{\prime}}, \bar{a}_{11} v_{y}\left(x_{1}, y\right)$ is continuous acrose $\tau=\partial K \cap \partial K^{\prime}=P_{1} P_{4}$, we obtain

$$
F(\tau \cap \partial K)+F\left(\tau \cap \partial K^{\prime}\right)=\bar{a}_{11} \frac{h_{y}^{3}}{24} v_{y}\left(x_{1}, y\right)\left(\left(u_{x y}\right)^{c}(K)-\left(u_{x y}\right)^{c}\left(K^{\prime}\right)\right) .
$$

Let $i_{h} w \in C^{0}(\Omega),\left.i_{h} w\right|_{K} \in Q_{1}(K)$, be an approximation of function $w$, which satisfies

$$
\left\|w-i_{h} w\right\|_{0, p} \leq C h\|w\|_{1, p}, 2 \leq p \leq \infty .
$$

For example, for $p=2$ we may take $i_{h} w=P_{h} w, P_{h}$ is the $L_{2}$-projection operator; For $p>2$, we may take $i_{h} w=\Pi_{h} w$. Then, from (3.15) and the inverse inequality, we have

$$
\begin{aligned}
& F(\tau \cap \partial K)+F\left(\tau \cap \partial K^{\prime}\right) \\
& =\bar{a}_{11} \frac{h_{y}^{3}}{24} v_{y}\left(x_{1}, y\right)\left(\left(u_{x y}\right)^{c}(K)-i_{h} u_{x y}(M)+i_{h} u_{x y}(M)-\left(u_{x y}\right)^{c}\left(K^{\prime}\right)\right) \\
& \leq C h_{y}^{3} h_{K}^{-\frac{2}{q}}\|v\|_{1, q, K}\left(h_{K}^{-\frac{2}{p}}\left\|\left(u_{x y}\right)^{c}-i_{h} u_{x y}\right\|_{0, p, K}+h_{K^{\prime}}^{-\frac{2}{p}}\left\|\left(u_{x y}\right)^{c}-i_{h} u_{x y}\right\|_{0, p, K^{\prime}}\right) \\
& \leq C h_{y}\|v\|_{1, q, K}\left(\left\|\left(u_{x y}\right)^{c}-u_{x y}\right\|_{0, p, K \cup K^{\prime}}+\left\|u_{x y}-i_{h} u_{x y}\right\|_{0, p, K \cup K^{\prime}}\right),
\end{aligned}
$$

where we have used the fact that $h_{y}=\left|\partial K \bigcap \partial K^{\prime}\right| \leq \min \left\{h_{K}, h_{K^{\prime}}\right\}$. Combining (3.13) with (3.16), we can conclude that

$$
E_{2} \leq C h\|v\|_{1, q}\left(\left\|u_{x y}-\left(u_{x y}\right)^{c}\right\|_{0, p}+\left\|u_{x y}-i_{h} u_{x y}\right\|_{0, p}\right) \leq C h^{2}\|u\|_{3, p}\|v\|_{1, q} .
$$

Substituting estimates $E_{1} \sim E_{4}$ into (3.9), we complete the proof by using (3.1).

From Theorem 3.1, we immediately obtain the following superclose result.

Theorem 3.2 Let $u$ and $u_{h}$ be the solutions of problem (1.5) and (2.8), respectively, $u \in H^{3}(\Omega)$. Then we have

$$
\left\|\Pi_{h} u-u_{h}\right\|_{1} \leq C h^{2}\|u\|_{3} .
$$


Proof From Lemma 2.2, error equation (2.9) and weak estimate (3.8), we have

$$
\begin{aligned}
& C\left\|u_{h}-\Pi_{h} u\right\|_{1}^{2} \leq a_{h}\left(u_{h}-\Pi_{h} u, \Pi_{h}^{*}\left(u_{h}-\Pi_{h} u\right)\right) \\
& =a_{h}\left(u-\Pi_{h} u, \Pi_{h}^{*}\left(u_{h}-\Pi_{h} u\right)\right) \leq C h^{2}\|u\|_{3}\left\|u_{h}-\Pi_{h} u\right\|_{1} .
\end{aligned}
$$

This gives the conclusion.

By using the embedding theory and weak embedding inequality of finite element space [21], a direct result from Theorem 3.2 is the following optimal $L_{p}$-error estimates,

$$
\begin{aligned}
& \left\|u-u_{h}\right\|_{0, p} \leq\left\|u-\Pi_{h} u\right\|_{0, p}+\left\|\Pi_{h} u-u_{h}\right\|_{0, p} \leq C_{p} h^{2}\left(\|u\|_{2, p}+\|u\|_{3}\right), 1<p<\infty \\
& \left\|u-u_{h}\right\|_{0, \infty} \leq C\left(h^{2}\|u\|_{2, \infty}+|\ln h|^{\frac{1}{2}}\left\|\Pi_{h} u-u_{h}\right\|_{1}\right) \leq C h^{2}\left(\|u\|_{2, \infty}+|\ln h|^{\frac{1}{2}}\|u\|_{3}\right) .
\end{aligned}
$$

\section{Piecewise-point superconvergence for gradient approximation}

Definition 4.1 Let point $P \in \Omega$, we call $P$ an optimal stress point for interpolation operator $\Pi_{h}$, if

$$
\left|\left(\nabla u-\bar{\nabla} \Pi_{h} u\right)(P)\right| \leq C h^{2}\|u\|_{3, \infty, E},
$$

where $E$ is the union of elements containing point $P$ and $\bar{\nabla} w(P)$ denotes the average value of gradient $\nabla w(P)$ in elements containing point $P$.

It is well known that for the bilinear interpolation $\Pi_{h} u$ on rectangular meshes, the optimal stress point set $S$ is composed of all interior mesh points, midpoints of interior edges and the midpoints of elements [16,21]. In this section, we will derive the $W^{1, \infty}$-superconvergence result for the bilinear finite volume solution $u_{h}$ on set $S$.

We first give some lemmas. Let rectangular element $K=\square P_{1} P_{2} P_{3} P_{4}$. For $w_{h} \in U_{h}$, set $w_{i}=w\left(P_{i}\right), i=1, \cdots, 4$. Introduce the discrete $H^{1}$-norm by

$$
\left|w_{h}\right|_{1, h}=\left(\sum_{K \in T_{h}}\left|w_{h}\right|_{1, h, K}^{2}\right)^{\frac{1}{2}}, w_{h} \in U_{h}
$$

where

$$
\left|w_{h}\right|_{1, h, K}^{2}=\left(w_{2}-w_{1}\right)^{2}+\left(w_{3}-w_{2}\right)^{2}+\left(w_{3}-w_{4}\right)^{2}+\left(w_{4}-w_{1}\right)^{2} .
$$


Lemma 4.1 The discrete norm $\left|w_{h}\right|_{1, h}$ is equivalent to the norm $\left|w_{h}\right|_{1}$ on space $U_{h}$, and

$$
\frac{1}{6 \gamma}\left|w_{h}\right|_{1, h, K}^{2} \leq\left|w_{h}\right|_{1, K}^{2} \leq \frac{\gamma}{2}\left|w_{h}\right|_{1, h, K}^{2}, \forall K \in T_{h}
$$

Proof Let $K=\square P_{1} P_{2} P_{3} P_{4}=\left[x_{1}, x_{1}+h_{x}\right] \times\left[y_{1}, y_{1}+h_{y}\right], \xi=\left(x-x_{1}\right) / h_{x}, \eta=(y-$ $\left.y_{1}\right) / h_{y}$. For $w_{h} \in Q_{1}(K)$, we may write

$$
w_{h}(x, y)=w_{1}+\left(w_{2}-w_{1}\right) \xi+\left(w_{4}-w_{1}\right) \eta+\left(w_{3}+w_{1}-w_{2}-w_{4}\right) \xi \eta .
$$

Set

$$
\begin{aligned}
& w_{21}=w_{2}-w_{1}, w_{41}=w_{4}-w_{1}, w_{32}=w_{3}-w_{2}, w_{34}=w_{3}-w_{4}, \\
& w_{1234}=w_{3}+w_{1}-w_{2}-w_{4}=w_{34}-w_{21}=w_{32}-w_{41} .
\end{aligned}
$$

Then, we have from (4.5)-4.7) that

$$
\begin{aligned}
\left|w_{h}\right|_{1, K}^{2} & =\int_{K}\left|\nabla w_{h}\right|^{2} d x d y=\int_{0}^{1} \int_{0}^{1}\left(\left(\partial_{\xi} w_{h} / h_{x}\right)^{2}+\left(\partial_{\eta} w_{h} / h_{y}\right)^{2}\right) h_{x} h_{y} d \xi d \eta \\
& =\frac{h_{y}}{h_{x}} \int_{0}^{1} \int_{0}^{1}\left(w_{21}+w_{1234} \eta\right)^{2} d \xi d \eta+\frac{h_{x}}{h_{y}} \int_{0}^{1} \int_{0}^{1}\left(w_{41}+w_{1234} \xi\right)^{2} d \xi d \eta \\
& =\frac{h_{y}}{h_{x}}\left(w_{21}^{2}+w_{21} w_{1234}+\frac{1}{3} w_{1234}^{2}\right)+\frac{h_{x}}{h_{y}}\left(w_{41}^{2}+w_{41} w_{1234}+\frac{1}{3} w_{1234}^{2}\right) \\
& =\frac{1}{3} \frac{h_{y}}{h_{x}}\left(w_{21}^{2}+w_{21} w_{34}+w_{34}^{2}\right)+\frac{1}{3} \frac{h_{x}}{h_{y}}\left(w_{32}^{2}+w_{32} w_{41}+w_{41}^{2}\right) .
\end{aligned}
$$

Hence, it follows from the Cauchy inequality and the regularity condition (2.2) that

$$
\frac{1}{6 \gamma}\left(w_{21}^{2}+w_{34}^{2}+w_{32}^{2}+w_{41}^{2}\right) \leq\left|w_{h}\right|_{1, K}^{2} \leq \frac{\gamma}{2}\left(w_{21}^{2}+w_{34}^{2}+w_{32}^{2}+w_{41}^{2}\right) .
$$

Combining this with (4.3), the proof is completed.

Let $A^{c}=\left(a_{i j}^{c}\right)_{2 \times 2}$ be the piecewise constant approximation of matrix function $A$.

Lemma 4.2 The following identity holds for $w, v \in U_{h}$,

$$
\int_{\partial K} n \cdot\left(A^{c} \nabla w\right)\left(\Pi_{h}^{*} v-v\right) d s=\frac{h_{y}^{3} h_{x}}{24} a_{11}^{c} v_{x y} w_{x y}+\frac{h_{y} h_{x}^{3}}{24} a_{22}^{c} v_{x y} w_{x y} .
$$


Proof From Lemma 3.2 we obtain

$$
\begin{aligned}
& \int_{\partial K} n \cdot\left(A^{c} \nabla w\right)\left(\Pi_{h}^{*} v-v\right) d s \\
= & \int_{P_{2} P_{3}}\left(\Pi_{h}^{*} v-v\right)\left(a_{11}^{c} w_{x}+a_{12}^{c} w_{y}\right) d y-\int_{P_{1} P_{4}}\left(\Pi_{h}^{*} v-v\right)\left(a_{11}^{c} w_{x}+a_{12}^{c} w_{y}\right) d y \\
& +\int_{P_{4} P_{3}}\left(\Pi_{h}^{*} v-v\right)\left(a_{21}^{c} w_{x}+a_{22}^{c} w_{y}\right) d x-\int_{P_{1} P_{2}}\left(\Pi_{h}^{*} v-v\right)\left(a_{21}^{c} w_{x}+a_{22}^{c} w_{y}\right) d x \\
= & \frac{h_{y}^{3}}{24} a_{11}^{c} v_{y}\left(x_{2}, y\right) w_{x y}-\frac{h_{y}^{3}}{24} a_{11}^{c} v_{y}\left(x_{1}, y\right) w_{x y} \\
& +\frac{h_{x}^{3}}{24} a_{22}^{c} v_{x}\left(x, y_{2}\right) w_{x y}-\frac{h_{x}^{3}}{24} a_{22}^{c} v_{x}\left(x, y_{1}\right) w_{x y} \\
= & \frac{h_{y}^{3} h_{x}}{24} a_{11}^{c} v_{x y} w_{x y}+\frac{h_{y} h_{x}^{3}}{24} a_{22}^{c} v_{x y} w_{x y} .
\end{aligned}
$$

Now, we need to introduce the regularized Green function [19,21]. For any given $z \in \Omega$, let $\delta_{h}^{z} \in U_{h}$ be the smooth $\delta$-function which satisfies,

$$
\left(\delta_{h}^{z}, v_{h}\right)=v_{h}(z), z \in \Omega, \forall v_{h} \in U_{h}
$$

For any appointed direction $L$, define the direction derivative

$$
\partial_{z} v(z)=\lim _{\Delta z \rightarrow 0, \Delta z / / L}(v(z+\Delta z)-v(z)) /|\Delta z|
$$

Then, there exists a regularized Green function of derivative type $\partial_{z} G^{z}(x) \in H_{0}^{1}(\Omega) \bigcap H^{2}(\Omega)$ such that

$$
a\left(v, \partial_{z} G^{z}\right)=\left(\partial_{z} \delta_{h}^{z}, v\right), \forall v \in H_{0}^{1}(\Omega)
$$

Let $\partial_{z} G_{h}^{z} \in U_{h}$ be the finite element approximation of $\partial_{z} G^{z}$ such that

$$
a\left(v_{h}, \partial_{z} G^{z}-\partial_{z} G_{h}^{z}\right)=0, \forall v_{h} \in U_{h}
$$

Clearly, we have

$$
a\left(v_{h}, \partial_{z} G_{h}^{z}\right)=a\left(v_{h}, \partial_{z} G^{z}\right)=\left(\partial_{z} \delta_{h}^{z}, v_{h}\right)=\partial_{z} v_{h}(z), \forall v_{h} \in U_{h}
$$

Assume that partition $T_{h}$ is quasi-uniform so that the inverse inequality holds on finite element space $U_{h}$. Under this condition, the following boundness estimates were given in [19,21]

$$
\left\|\partial_{z} G_{h}^{z}\right\|_{1} \leq C h^{-1}|\ln h|^{\frac{1}{2}}, \quad\left\|\partial_{z} G_{h}^{z}\right\|_{1,1} \leq C|\ln h|
$$


where $C$ is a positive constant independent of $z \in \Omega$.

Theorem 4.1 Let partition $T_{h}$ be quasi-uniform, and $u$ and $u_{h}$ be the solutions of problem (1.5) and (2.8), respectively, $u \in W^{3, \infty}(\Omega)$. Then we have

$$
\left\|\Pi_{h} u-u_{h}\right\|_{1, \infty} \leq C h^{2}|\ln h|\|u\|_{3, \infty} .
$$

Proof From (4.9), error equation 2.9), Theorem 3.1 and (4.10), we obtain

$$
\begin{aligned}
& \partial_{z}\left(u_{h}-\Pi_{h} u\right)(z)=a\left(u_{h}-\Pi_{h} u, \partial_{z} G_{h}^{z}\right) \\
& =a\left(u_{h}-\Pi_{h} u, \partial_{z} G_{h}^{z}\right)-a_{h}\left(u_{h}-\Pi_{h} u, \Pi_{h}^{*} \partial_{z} G_{h}^{z}\right)+a_{h}\left(u_{h}-\Pi_{h} u, \Pi_{h}^{*} \partial_{z} G_{h}^{z}\right) \\
& =a\left(u_{h}-\Pi_{h} u, \partial_{z} G_{h}^{z}\right)-a_{h}\left(u_{h}-\Pi_{h} u, \Pi_{h}^{*} \partial_{z} G_{h}^{z}\right)+a_{h}\left(u-\Pi_{h} u, \Pi_{h}^{*} \partial_{z} G_{h}^{z}\right) \\
& \leq a\left(u_{h}-\Pi_{h} u, \partial_{z} G_{h}^{z}\right)-a_{h}\left(u_{h}-\Pi_{h} u, \Pi_{h}^{*} \partial_{z} G_{h}^{z}\right)+C h^{2}\|u\|_{3, \infty}\left\|\partial_{z} G_{h}^{z}\right\|_{1,1} \\
& \leq a\left(u_{h}-\Pi_{h} u, \partial_{z} G_{h}^{z}\right)-a_{h}\left(u_{h}-\Pi_{h} u, \Pi_{h}^{*} \partial_{z} G_{h}^{z}\right)+C h^{2}|\ln h|\|u\|_{3, \infty}
\end{aligned}
$$

Below we need to estimate (see Lemma 3.1)

$$
\begin{aligned}
& a\left(u_{h}-\Pi_{h} u, \partial_{z} G_{h}^{z}\right)-a_{h}\left(u_{h}-\Pi_{h} u, \Pi_{h}^{*} \partial_{z} G_{h}^{z}\right) \\
= & \sum_{K \in T_{h}} \int_{\partial K} n \cdot\left(A-A^{c}\right) \nabla\left(u_{h}-\Pi_{h} u\right)\left(\partial_{z} G_{h}^{z}-\Pi_{h}^{*} \partial_{z} G_{h}^{z}\right) d s \\
& +\sum_{K \in T_{h}} \int_{\partial K} n \cdot A^{c} \nabla\left(u_{h}-\Pi_{h} u\right)\left(\partial_{z} G_{h}^{z}-\Pi_{h}^{*} \partial_{z} G_{h}^{z}\right) d s \\
& +\sum_{K \in T_{h}}\left(-\operatorname{div}\left(A \nabla\left(u_{h}-\Pi_{h} u\right)\right), \partial_{z} G_{h}^{z}-\Pi_{h}^{*} \partial_{z} G_{h}^{z}\right)_{K} \\
& +\left(c\left(u_{h}-\Pi_{h} u\right), \partial_{z} G_{h}^{z}-\Pi_{h}^{*} \partial_{z} G_{h}^{z}\right)_{K} \\
= & S_{1}+S_{2}+S_{3}+S_{4} .
\end{aligned}
$$

First, using (2.11), 2.12) and 2.15), Theorem 3.2 and (4.10), we have

$$
\begin{aligned}
S_{1} & \leq C \sum_{K \in T_{h}} h_{K}|A|_{1, \infty}\left\|\nabla\left(u_{h}-\Pi_{h} u\right)\right\|_{0, \partial K}\left\|\partial_{z} G_{h}^{z}-\Pi_{h}^{*} \partial_{z} G_{h}^{z}\right\|_{0, \partial K} \\
& \leq C h^{\frac{1}{2}}\left\|\nabla\left(u_{h}-\Pi_{h} u\right)\right\| h^{\frac{1}{2}}\left\|\partial_{z} G_{h}^{z}\right\|_{1} \\
& \leq C h^{3}\|u\|_{3}\left\|\partial_{z} G_{h}^{z}\right\|_{1} \leq C h^{2}|\ln h|^{\frac{1}{2}}\|u\|_{3} .
\end{aligned}
$$

Next, it follows from Lemma 4.2 and inverse inequality (2.12) that

$$
\begin{aligned}
S_{2} & =\leq \sum_{K \in T_{h}}|A|_{\infty} \frac{h_{K}^{4}}{12}\left|\left(u_{h}-\Pi_{h} u\right)_{x y}\right|_{0, \infty, K}\left|\left(\partial_{z} G_{h}^{z}\right)_{x y}\right|_{0, \infty, K} \\
& \leq C \sum_{K \in T_{h}} h_{K}^{2}\left|\left(u_{h}-\Pi_{h} u\right)_{x y}\right|_{0, \infty, K}\left\|\partial_{z} G_{h}^{z}\right\|_{1, K} .
\end{aligned}
$$


From 4.5) and Lemma 4.1, we have

$$
\left|\left(u_{h}-\Pi_{h} u\right)_{x y}\right|=\left|\left(u_{h}-\Pi_{h} u\right)_{1234}\right| \leq \sqrt{2}\left|u_{h}-\Pi_{h} u\right|_{1, K, h} \leq \sqrt{12 \gamma}\left|u_{h}-\Pi_{h} u\right|_{1, K}
$$

Substituting this estimate into $S_{2}$ and using Theorem 3.2, we obtain

$$
S_{2} \leq C h^{2}\left\|u_{h}-\Pi_{h} u\right\|_{1}\left\|\partial_{z} G_{h}^{z}\right\|_{1} \leq C h^{3}|\ln h|^{\frac{1}{2}}\|u\|_{3}
$$

Similarly, by Lemma 4.1 and Theorem 3.2, we have (noting that $\left(u_{h}-\Pi_{h} u\right)_{x x}=$ $\left.\left(u_{h}-\Pi_{h} u\right)_{y y}=0\right)$

$$
\begin{aligned}
S_{3} & \left.\leq C \sum_{K \in T_{h}}\left(\left\|\nabla\left(u_{h}-\Pi_{h} u\right)\right\|_{0, K}+\left\|\left(u_{h}-\Pi_{h} u\right)_{x y}\right\|_{0, K}\right) \| \partial_{z} G_{h}^{z}-\Pi_{h}^{*} \partial_{z} G_{h}^{z}\right) \|_{0, K} \\
& \left.\leq C \sum_{K \in T_{h}}\left\|u_{h}-\Pi_{h} u\right\|_{1, K} \| \partial_{z} G_{h}^{z}-\Pi_{h}^{*} \partial_{z} G_{h}^{z}\right) \|_{0, K} \\
& \leq C h^{3}\|u\|_{3}\left\|\partial_{z} G_{h}^{z}\right\|_{1} \leq C h^{2}|\ln h|^{\frac{1}{2}}\|u\|_{3} .
\end{aligned}
$$

For $S_{4}$, using Theorem 3.2, 2.14) and (4.10) to obtain

$$
\begin{aligned}
S_{4} & \leq C \sum_{K \in T_{h}}|A|_{1, \infty}\left\|u_{h}-\Pi_{h} u\right\|_{1, K}\left\|\partial_{z} G_{h}^{z}-\Pi_{h}^{*} \partial_{z} G_{h}^{z}\right\|_{0, K} \\
& \leq C h^{2}\|u\|_{3} h\left\|\partial_{z} G_{h}^{z}\right\|_{1} \leq C h^{2}|\ln h|^{\frac{1}{2}}\|u\|_{3} .
\end{aligned}
$$

Substituting estimates $S_{1} \sim S_{4}$ into (4.13), we complete the proof by (4.12).

Now we can give the main superconvergence result.

Theorem 4.2 Let partition $T_{h}$ be quasi-uniform, and $u$ and $u_{h}$ be the solutions of problem (1.5) and (2.8), respectively, $u \in W^{3, \infty}(\Omega)$. Then we have

$$
\max _{P \in S}\left|\left(\nabla u-\bar{\nabla} u_{h}\right)(P)\right| \leq C h^{2}|\ln h|\|u\|_{3, \infty}
$$

where $\bar{\nabla} w(P)$ denotes the average values of gradient $\nabla w(P)$ in elements containing point $P$ and $S$ is the optima stress point set composed of all interior mesh points, the midpoints of interior edges and the midpoints of elements.

Proof From 4.1 and Theorem 4.1, we immediately obtain the conclusion by using the triangle inequality. 


\section{Numerical example}

In this section, we will present some numerical results to illustrate the theoretical analysis.

Let us consider problem 1.5 with the data:

$$
A(x, y)=\left(\begin{array}{cc}
e^{2 x}+y^{3}+1 & e^{x+y} \\
e^{x+y} & e^{2 y}+x^{3}+1
\end{array}\right), \quad c(x, y)=2+x+y .
$$

We take $\Omega=[0,1]^{2}$ and the exact solution $u(x, y)=2 \sin (2 \pi x) \sin (3 \pi y)$.

In the numerical experiments, we first partition domain $\Omega$ into a rectangular mesh $T_{h}$, then the refined meshes are obtained by connecting the midpoints of each edge of elements in $T_{h}$. Thus, a rectangular mesh sequence is generated with successively halving mesh size $h / 2^{i}, i=1,2, \cdots$.

Denote by $e_{h}=\max _{P \in S}\left|\nabla u(P)-\bar{\nabla} u_{h}(P)\right|$ the computation error and the numerical convergence rate is computed by using the formula $r=\ln \left(e_{h} / e_{h / 2}\right) / \ln 2$. Table 1 gives the error and convergence rate with successively halved mesh sizes. We see that the convergence rate is just about $O\left(h^{2}\right)$, as the theoretical prediction.

Table 1 Convergence rate of gradient approximation on set $S$

\begin{tabular}{ccccccc}
\hline$h$ & $1 / 4$ & $1 / 8$ & $1 / 16$ & $1 / 32$ & $1 / 64$ & $1 / 128$ \\
\hline$e_{h}$ & 1.212 & $3.099 \mathrm{e}-1$ & $7.856 \mathrm{e}-2$ & $1.969 \mathrm{e}-2$ & $4.949 \mathrm{e}-3$ & $1.243 \mathrm{e}-3$ \\
rate & - & 1.9671 & 1.9802 & 1.9961 & 1.9926 & 1.9932 \\
\hline
\end{tabular}

\section{References}

1. Cai, Z.Q.: On the finite volume element method. Numer. Math. 58, 713-735 (1991)

2. Chen, Z.Y.: $L^{2}$ estimate of linear element generalized difference schemes. Acta. Sci. Nat. Univ. Sunyatseni 33, 22-28 (1994)

3. Chen, Z.Y.: Superconvergence of generalized difference methods for elliptic boundary value problem. Numer. Math. A J. of Chinese Univ.(English Ser) 3, 163-171 (1994)

4. Chen, Z.Y., Li, R.H., Zhou, A.H.: A note on the optimal $L^{2}$-estimate of the finite volume element method. Adv. Comput. Math. 16, 291-303 (2002) 
5. Chen, L.: A new class of high order finite volume element methods for second order elliptic equations. SIAM J. Numer. Anal. 47, 4021-4023 (2010)

6. Chou, S.H., Li, Q.: Error estimates in $L^{2}, H^{1}, L^{\infty}$ in covolume methods for elliptic and parabolic problem: a unified approach. Math. Comp. 69, 103-120 (2000)

7. Chou, S.H., Ye, X.: Superconvergence of finite volume methods for the second order elliptic problem. Comput. Methods Appl. Mech. Engrg. 196, 3706-3712 (2007)

8. Ewing, R.E., Lin, T., Lin, Y.P.: On the accuracy of the finite volume element method based on piecewise linear polynomials. SIAM J. Numer. Anal. 39, 1865-1888 (2002)

9. Lazarov, R., Michev, I., Vassilevski, P.: Finite volume methods for convection-diffusion problems. SIAM J. Numer. Anal. 33, 31-55 (1996)

10. Li, R.H., Chen, Z.Y., Wu, W.: Generalized difference methods for differential equations: numerical analysis of finite volume methods. Marcel, New York (2000)

11. Lin, Q., Zhu, Q.D.: The preprocessing and postprocessing for the finite element methods. Shanghai Sci \& Tech Publishing, Shanghai (1994)

12. Lv, J.L., Li, Y.H.: $L^{2}$ error estimates and superconvergence of the finite volume element methods on quadrilateral meshes. Adv. Comput. Math. 37, 393-416 (2012)

13. Süli, E.: Convergence of finite volume schemes for poission's equation on nonuniform meshes. SIAM J. Numer. Anal. 28, 1419-1430 (1991)

14. Wu, H.J., Li, R.H.: Error estimate for finite volume element methods for general second order elliptic problem. Numer. Meth. PDEs. 19, 693-708 (2003)

15. Xu, J.C., Zou, Q.S.: Analysis of linear and quadratic simplical finite volume methods for elliptic equations. Numer. Math. 111, 469-492 (2009)

16. Zhang, L., Li, L.K.: On superconvergence of isopareametric bilienar finite elements. Comm. Numer. Meth. Enrg. 12, 849-862 (1996)

17. Zhang, T., Lin, Y.P., Tait, R.J.: On the finite volume element version of Ritz-Volterra projection and applications to related equations. J. Comp. Math. 20, 491-504 (2002)

18. Zhang T., Sheng Y.: Superconvergence and Gradient Recovery for a Finite Volume Element Method for Solving Convection-Diffusion Equations. Numer. Meth. PDEs. 30, 1152-1168 (2014)

19. Zhang, T.: Finite element methods for partial differential-integral equations. Science Press, Beijing (2009)

20. Zhang, Z.M., Zou, Q.S.: Some recent advances on vertex centered finite volume element methods for elliptic equations. Sci. China Mathematics 56, 2507-2522 (2013)

21. Zhu, Q.D., Lin, Q.: The superconvergence theory of finite elements. Hunan Science and Technology Publishing House, Changsha (1989) 